\title{
ON A FORMALISM WHICH MAKES ANY SEQUENCE OF SYMBOLS WELL-FORMED
}

\author{
SHIGEO ÖHAMA
}

Any finite sequence of primitive symbols is not always well-formed in the usual formalisms. But in a certain formal system, we can normalize any sequence of symbols uniquely so that it becomes well-formed. An example of this kind has been introduced by Ono [2]. While we were drawing up a practical programming along Ono's line, we attained another system, a modification of his system. The purpose of the present paper is to introduce this modified system and its application. In 1, we will describe a method of normalizing sentences in $\boldsymbol{L O}^{1)}$ having only two logical constants, implication and universal quantifier, so that any finite sequence of symbols becomes wellformed. In 2, we will show an application of 1 to proof. I wish to express my appreciation to Prof. K. Ono for his significant suggestions and advices.

1. Normalizing sentences. In our formalism, similarly in Ono [2], we use only one category of variables and a pair of brackets "[" and "]" called HEAD- and TAIL-BRACKET, respectively. So a sentence $\mathscr{A}$ in usual notation is transformed to $A$ as follows;

(1) If $\mathscr{A}$ is an $n$-ary relation $R(x,---, z)$, then $A$ is $[r x---z]$, where $r$ is denoted as a predicate variable corresponding to $R$,

(2) If $\mathscr{A}$ is of the form $(x)---(z) \mathscr{B}$, then $A$ is of the form $x---z B$,

(3) If $\mathscr{A}$ is of the form $\mathscr{B} \rightarrow(--(\mathscr{C} \rightarrow \mathscr{D})---)$ and $B, C, D$ are translated forms of $\mathscr{B}, \mathscr{C}, \mathscr{D}$, respectively, then $A$ is of the form $B^{*} \ldots$ $C^{*} D^{*}$, where $B^{*}$ and $C^{*}$ denote $B$ and $C$, respectively, in the case of the left most symbols of $B$ and $C$ being corresponding head-brackets of the right most tail-brackets of them and otherwise $[B]$ and $[C]$, respectively, and $D^{*}$ denote $[D]$ in the case of the left most symbol of $D$ being a variable and otherwise $D$.

Received October 21, 1966.

1) See Ono [ 1$]$. 
For example, a sentence

$$
(x)(R(x, u) \rightarrow(y)(z)(S(y, z, u) \rightarrow R(y, z))) \rightarrow(R(w, u) \rightarrow S(w, v, u)),
$$

where $R$ and $S$ are binary and ternary relation, respectively, is translated as follows,

$$
[x[r x u][y z[s y z u][r y z]]][r w u][s w v u] .
$$

Now, let us define SENTENCE and NORMAL SENTENCE.

Definition 1. Any sequence of symbols is called SENTENCE.

Definition 2. Any sentence $A$ is called NORMAL SENTENCE if and only if

(1) A includes at least one barcket,

(2) any tail-bracket in $A$ is not immediately followed by a variable(s),

(3) in any segment $A_{i}$ (i.e. subsequence of $A$, from the first symbol to the $i$-th symbol), the number of tail-brackets does not exceed the number of head-brackets and the whole number of tail-brackets is equal to that of head-brackets.

We can uniquely normalize any sentence, if not normal, by the following operation.

Operation 1. If a given sentence does not satisfy the condition (2) in Definition 2 , insert a head-bracket between the tail-bracket and the variable(s), and repeat this operation until a resulting sentence satisfies the condition (2) in Definition 2.

Operation 2. If the sentence resulting from Operation 1 does not satisfy the condition (1) or (3) in Definition 2, then add head-bracket(s) and tail-bracket(s) at the beginning and at the end of the sentence, respectively, so that it satisfies the condition (1) and (3) in Definition 2.

For example, a sentence

$$
f u v w]] x y z[g x y z u] f x y z]][x y z[g u v w z] f x y w
$$

becomes by Operation 1

$$
\left.f u v w]] \stackrel{\downarrow}{[x} x z[g x y z u]_{[}^{\downarrow}[f x y z]\right][x y z[g u v w z] \stackrel{\downarrow}{[} f x y w
$$

and this becomes normal by applying Operation 2

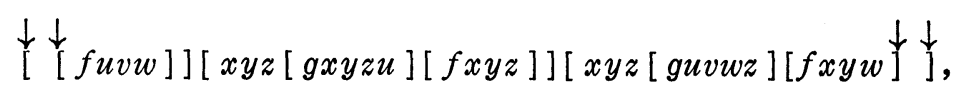


where $\stackrel{\downarrow}{\text { and }} \stackrel{\downarrow}{]}$ mean brackets added by each operation.

2. Application to PROOF. Our normalization excludes sentences of the form $x---z A u---w,{ }^{2)}$ which is regarded as normal sentence in Ono's system and is useful. But if we modify description of proof-notes a little, we can describe any proof-note without making use of sentences of the above form. Now let us rewrite the example proof in Ono [2] by our modified way.

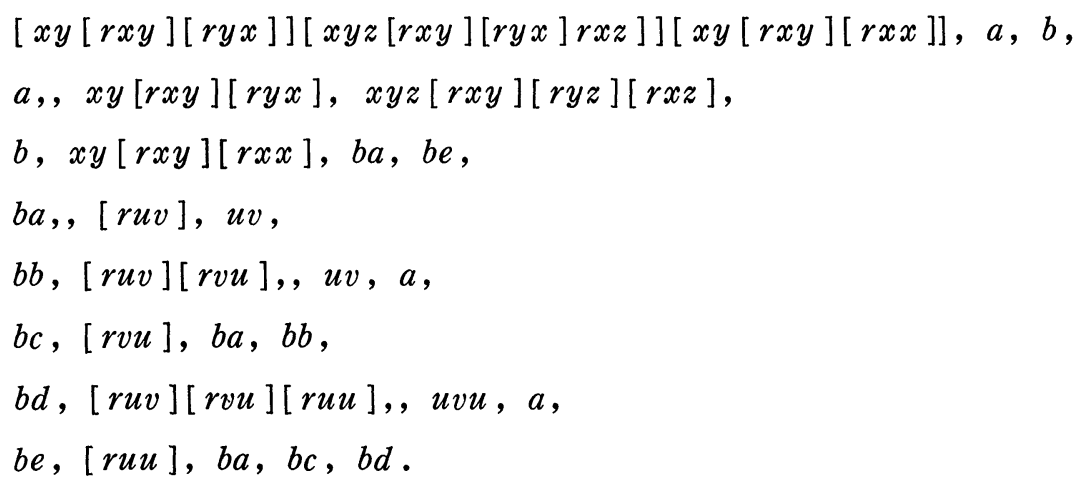

In assumption step $\varphi a$, we allow only one reference index which is a series of the same number of mutually distinct variables as the outest series of quantifiers in the step $\varphi$. And any variable of the reference index does not occur as free in any step beginning with $\varphi$ or beginning with an index in the ground of $\varphi$. The $\varphi a$ step

$$
\varphi a, \alpha_{1},---, \alpha_{k}, \sigma,
$$

means "Take any series of variables of fixed length, say $\sigma$, satisfying the condition $\alpha_{1},---, \alpha_{k}$ ". where $\alpha_{1},---, \alpha_{k}$ are sentences. ( $c f$. the step $\left.b a\right)$.

In assertion step $\varphi$, we allow the reference index following immediately after a sentence over two successive commas. The $\varphi$ step

$$
\varphi, \alpha, \sigma,---,
$$

means "Any series of variables of fixed length satisfies condition $\alpha$, so we take any one series of them and call it $\sigma$ ", where $\alpha$ is a sentance, $\sigma$ is an index, and "---" represents a sequence of indices $(c f$. steps $b b, b d)$."

2) For two variable series, $x---z$ and $u---w$ being the same length and a sentence $A$, this means that free variables $x,---, z$ in $A$ are substituted by $u,---, w$, respectively. 


\section{REFERENCES}

[1] Ono. K: A certain kind of formal theories, Nagoya Math. J., Vol. 25 (1965), pp. 59-86.

[2] - A formalism for primitive logic and mechanical proof-checking, Nagoya Math. J., Vol. 26 (1966), pp. 195-203.

Nagoya University 